\title{
Ontogênese do sistema subterrâneo de Pachyrhizus ahipa (Wedd.) Parodi (Fabaceae) ${ }^{1}$
}

\author{
CAMILLA R.D. MILANEZ2,3 e MARINA A. MORAES-DALLAQUA²
}

(recebido: 12 de fevereiro de 2003; aceito: 21 de agosto de 2003)

\begin{abstract}
Ontogeny of the subterranean system of Pachyrhizus ahipa (Wedd.) Parodi (Fabaceae)). A study on the ontogeny of the underground system of $P$. ahipa was held with the purpose of understanding both its morphology and its process of tuberization. Samples, collected from the hypocotyl region and from the primary root during different phases of plant development, were processed according to usual technic. The underground system of $P$. ahipa is composed by both a xylopodium and a tuberous root. The xylopodium is a cylindrical lignified region near the soil and gives rises to new sprouts. Immediately below to this part there is a primary tuberous root, which is predominantly fusiform. The root tuberization results from the activity of a typical vascular cambium and from accessory cambium originated from the parenchyma of the secondary xylem, after the first phase of the usual secondary growth. The xylopodium is of a mixed nature, as it arrises from the hypocotyl and from the base of the primary root.
\end{abstract}

Key words - Anatomy, Pachyrhizus, tuberous root, underground system, xylopodium

RESUMO - (Ontogênese do sistema subterrâneo de Pachyrhizus ahipa (Wedd.) Parodi (Fabaceae)). Realizou-se o estudo da ontogênese do sistema subterrâneo de P. ahipa com o objetivo de contribuir para o conhecimento da morfologia, bem como do processo de tuberização dos órgãos subterrâneos desta espécie. Amostras das regiões do hipocótilo e da raiz primária foram retiradas em diferentes fases de desenvolvimento da planta e processadas segundo técnica usual. Verificou-se que o sistema subterrâneo de $P$. ahipa compreende um xilopódio e uma raiz tuberosa. O xilopódio corresponde a uma porção cilíndrica, lenhosa e lignificada, próxima ao solo, da qual surgem novos brotos. Logo abaixo, encontra-se a raiz primária tuberosa, de formato predominantemente fusiforme. O processo de tuberização da raiz resulta da atividade de um câmbio vascular típico, juntamente com câmbios acessórios que surgem a partir da retomada de divisão celular do parênquima do xilema secundário, após a primeira fase do crescimento secundário usual. A ontogênese do xilopódio mostra que este apresenta natureza mista, formando-se a partir do crescimento secundário tanto do hipocótilo quanto da região basal da raiz primária tuberosa.

Palavras-chave - Anatomia, Pachyrhizus, raiz tuberosa, sistema subterrâneo, xilopódio

\section{Introdução}

Apesar da relevante importância dos sistemas subterrâneos quanto aos aspectos fisiológicos, ecofisiológicos e econômicos, os estudos morfoanatômicos envolvendo estas estruturas ainda são escassos, diante da grande diversidade encontrada nos diferentes ambientes.

Verifica-se, no entanto, que é vasta a terminologia utilizada para classificar sistemas subterrâneos, principalmente os que possuem natureza caulinar (Rocha 1995). Porém, apesar da variedade de termos, muitas vezes eles parecem insuficientes para designar o tipo de órgão subterrâneo que se quer descrever. Deste modo, torna-se necessário a realização de estudos com

\footnotetext{
1. Parte da Dissertação de Mestrado da primeira autora, Unesp Botucatu.

2. Univesidade Estadual Paulista, Instituto de Biociências, Departamento de Botânica, Caixa Postal 510, 18618-000 Botucatu, SP, Brasil.

3. Autora para correspondência: camillamilanez@bol.com.br
}

abordagem morfo-anatômica para a caracterização adequada dos diversos tipos de sistemas subterrâneos e correção de conceitos errôneos, que passaram a integrar a literatura científica.

De acordo com Lackey (1977), o gênero Pachyrhizus Rich. ex DC. pertence à família Fabaceae, tribo Phaseoleae, subtribo Diocleinae, e segundo Sørensen (1988) o gênero compreende cinco espécies das quais três são cultivadas [P. ahipa (Wedd.) Parodi, P. erosus Urban e P. tuberosus (Lam.) Spreng.] e duas $[P$. ferrugineus (Piper) M. Sørensen e P. panamensis R.T. Clausen] são selvagens.

Pachyrhizus é um dos poucos gêneros de leguminosas portadoras de raízes tuberosas comestíveis, apresentando crescente interesse devido ao reconhecimento do seu alto potencial de produção, de valor nutricional e da possibilidade do cultivo em larga escala de P. erosus, P. tuberosus e P. ahipa (Kay 1973, Sørensen et al. 1997).

Pachyrhizus ahipa caracteriza-se pelo acúmulo de amido de interesse industrial, em sua raiz tuberosa, e 
pela presença de rotenona, em suas sementes e folhas. A raiz é consumida quase exclusivamente crua, como uma fruta ou, ainda, cozida e sua casca desprende-se com bastante facilidade da porção interna, carnosa e branca (Grau 1997).

Os trabalhos envolvendo $P$. ahipa referem-se, principalmente, à morfologia da planta adulta (Sørensen 1988), aos aspectos agronômicos (Sørensen 1990), fisiológicos (Kjaer 1992) e à composição química da raiz tuberosa (Hansberry et al. 1947, Sørensen et al. 1997, Forsyth et al. 2002), não tendo sido realizado, até o momento, um estudo anatômico sobre o sistema subterrâneo desta espécie.

Com base nessas considerações, realizou-se o presente trabalho com o objetivo de contribuir para o conhecimento da morfologia e do processo de tuberização do sistema subterrâneo de Pachyrhizus ahipa.

\section{Material e métodos}

Sementes de Pachyrhizus ahipa (Wedd.) Parodi, fornecidas pelo Centro de Raízes e Amidos Tropicais (Cerat), Faculdade de Ciências Agronômicas, Fazenda Experimental Lageado, Botucatu, SP, foram selecionadas utilizando-se como parâmetro aquelas que apresentavam tamanhos médios e coloração homogênea.

Para a instalação do experimento, 100 sementes de Pachyrhizus ahipa foram previamente desinfectadas em hipoclorito de sódio a $20 \%$ da solução comercial, lavadas em água corrente e, posteriormente, colocadas em caixas "gerbox" sobre papel de filtro umedecido com água destilada, sendo mantidas em germinador modelo NT 705 a $25 \pm 1{ }^{\circ} \mathrm{C}$, sob iluminação fluorescente branca contínua. As plântulas, cinco dias após a germinação (apresentando cerca de $9 \mathrm{~cm}$ ), foram transplantadas para sacos de polietileno contendo uma mistura de terra e areia (1:1), sendo mantidas em estufa de sombrite $50 \%$ e regadas diariamente.

Outras 100 sementes foram semeadas diretamente em sacos de polietileno contendo substrato semelhante, sendo mantidas sob as condições descritas anteriormente. As sementes restantes foram semeadas em condições de campo, em virtude de se tratar de uma espécie não nativa ou cultivada no Brasil, desconhecendo-se as condições ideais de cultivo que pudessem interferir no estabelecimento e desenvolvimento do sistema subterrâneo da planta.

Das plantas obtidas, alguns exemplares foram prensados e registrados no Herbário "Irina D. Gemtchujnicov" (BOTU) do Departamento de Botânica do Instituto de Biociências da Unesp - Campus de Botucatu, sob o n. 23.130.

Para o estudo anatômico da ontogênese do sistema subterrâneo, foram retiradas amostras do hipocótilo e da raiz em diferentes fases de desenvolvimento. Os materiais vegetais amostrados foram fixados em FAA 50 (Johansen 1940), estocados em etanol $70 \%$ e, posteriormente, incluídos em historresina glicol-metacrilato, segundo técnica adotada por Guerrits (1964). Foram realizadas secções com auxílio de micrótomo rotativo, entre 7-10 $\mu \mathrm{m}$ de espessura, as quais foram submetidas à coloração com azul de toluidina $0,05 \%$ (O’Brien et al. 1964) e montadas em permount.

Os resultados foram documentados através de desenhos com auxílio de câmara clara, adaptada a esteriomicroscópio Wild e fotomicrografias, realizadas em fotomicroscópio Zeiss, modelo MC 80.

\section{Resultados}

Morfologia do sistema subterrâneo - O sistema subterrâneo de Pachyrhizus ahipa (figura 1) consiste de duas porções: um xilopódio, que se caracteriza como uma porção cilíndrica, lenhosa e lignificada, próxima ao solo e da qual surgem novos brotos (figuras 1,2); e uma porção tuberosa de formato predominantemente fusiforme (figura 1), que consiste na raiz primária tuberosa da planta, com casca de coloração amarronzada e que, internamente, possui inúmeros laticíferos, facilmente visíveis quando se destaca o revestimento (figura 3). Ocasionalmente, raízes laterais sofrem tuberização (figura 1).

A raiz primária não se tuberiza em toda a sua extensão, observando-se na região distal, uma redução no diâmetro que se mantém até o ápice do órgão (figura 1). Observam-se também nódulos radiculares na raiz primária e, em maior número, nas raízes laterais não tuberizadas.

Desenvolvimento da raiz primária - A raiz primária de Pachyrhizus ahipa com 7 dias (figuras 4-8), a 0,5 cm do ápice (figuras 4, 5), mostra apenas crescimento primário, com uma epiderme unisseriada, onde algumas células se diferenciam em pêlos radiculares.

O córtex constitui-se de 12 a 15 camadas de células parenquimáticas de formato isodiamétrico, com paredes delgadas e levemente onduladas, deixando entre si espaços intercelulares pequenos (figura 4); a endoderme é pouco evidente (figura 5).

O cilindro vascular compreende um periciclo plurisseriado, com células de vários formatos, verificando-se no centro, o desenvolvimento centrípeto incompleto do xilema primário (figura 5). A raiz é tetrarca, com quatro pólos de protoxilema alternados com floema (figura 5).

A aproximadamente 2,2 $\mathrm{cm}$ do ápice (figuras 6-8), ainda na região pilífera, observa-se a instalação de um câmbio típico e início de diferenciação de elementos de 

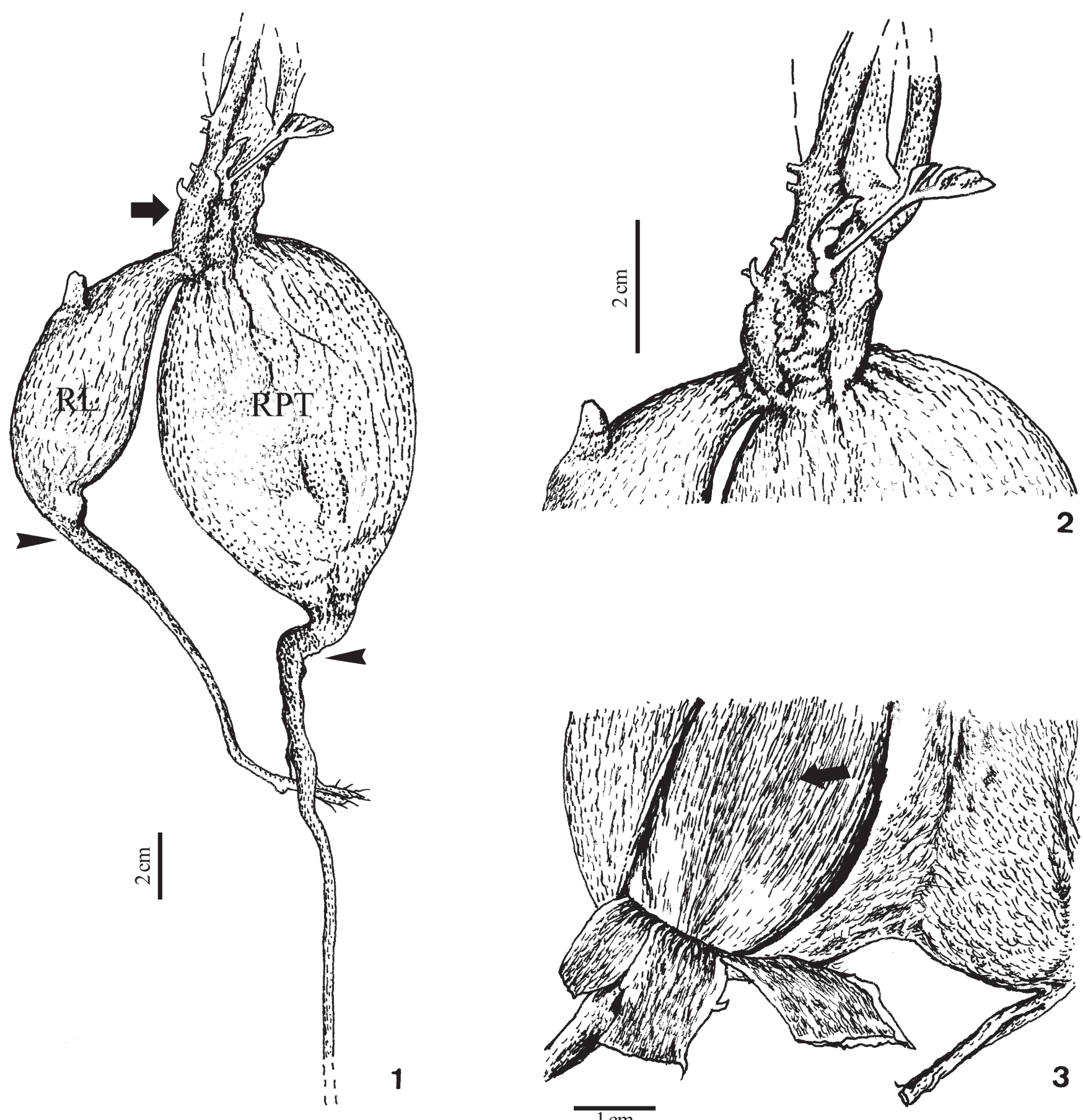

Figuras 1-3. Sistema subterrâneo da planta adulta de Pachyrhizus ahipa. 1. Aspecto geral, mostrando xilopódio (seta), raiz primária tuberosa (RPT) e raiz lateral (RL). Observar região não tuberizada das raízes tuberosas (pontas de seta). 2. Detalhe da figura anterior, evidenciando região do xilopódio com brotos. 3. Detalhe da região da "casca" da raiz tuberosa, mostrando laticífero (seta).

Figures 1-3. Subterranean system of adult plant of Pachyrhizus ahipa. 1. General view, showing xylopodium (arrow), primary tuberous root (RPT) and lateral root (RL). Notice non-tuberous root portions (arrow-heads). 2. Detail of previous figure, showing xylopodium with sprouts. 3. Detail of "bark" region of tuberous root, showing laticifers (arrow). 

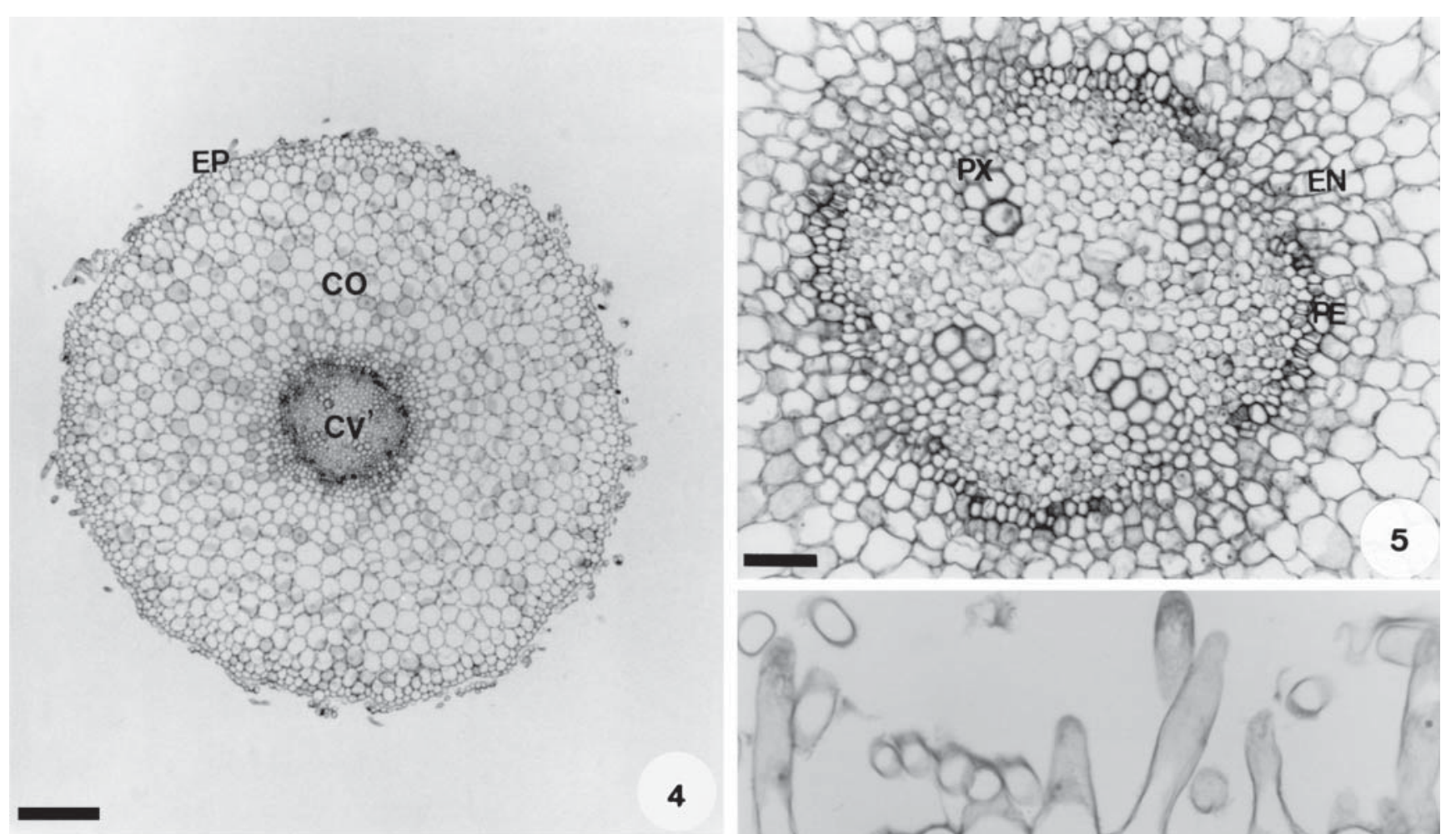

4
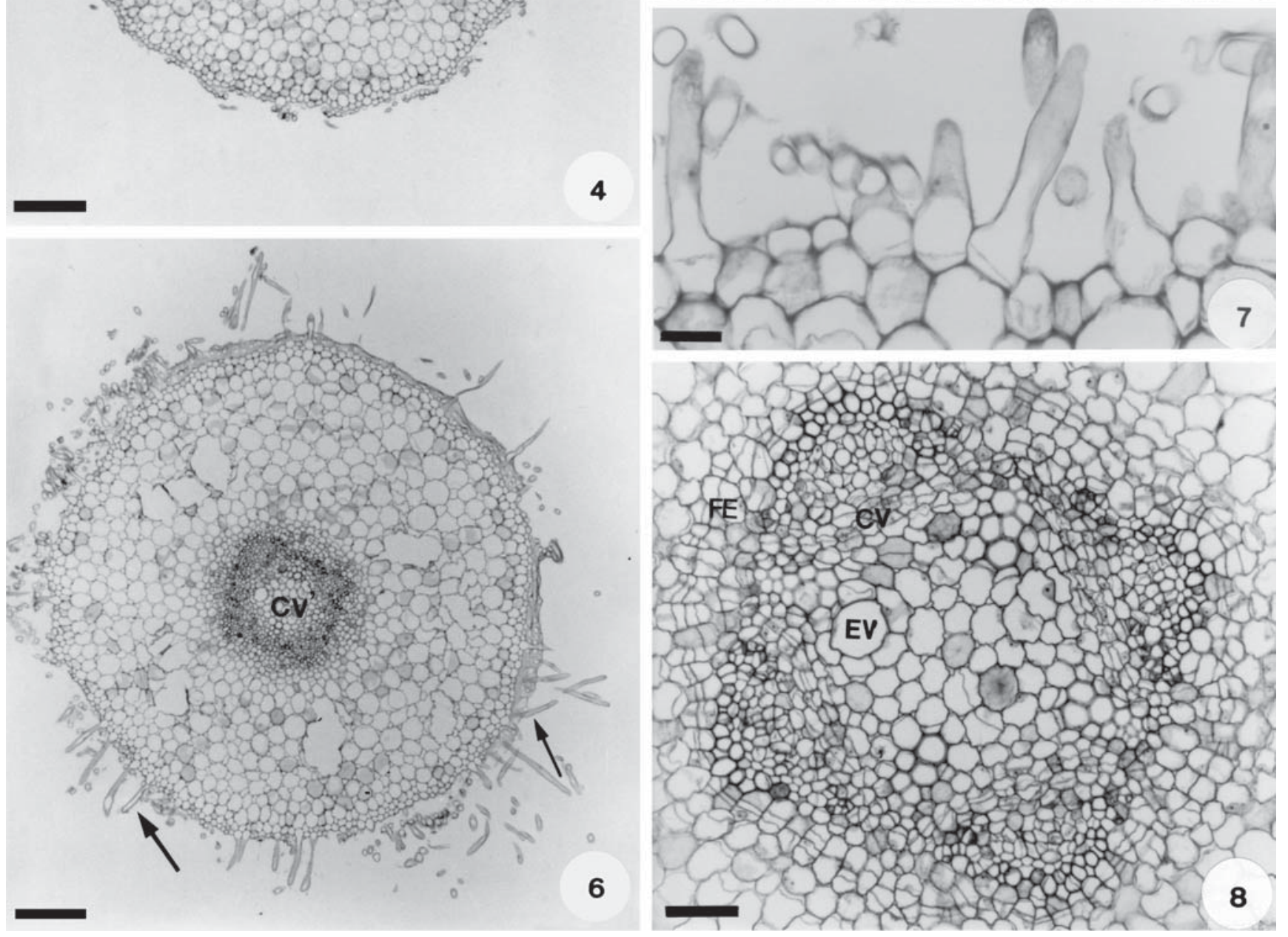

Figuras 4-8. Secções transversais medianas da raiz primária de Pachyrhizus ahipa com 7 dias. 4. A 0,5 cm do ápice. 5. Detalhe da figura anterior. 6. A $2,2 \mathrm{~cm}$ do ápice. 7. Detalhe de pêlos radiculares. 8. Detalhe da figura $6 .(\mathrm{CO}=$ córtex; $\mathrm{CV}=$ câmbio vascular; $\mathrm{CV}^{\prime}=$ cilindro vascular; $\mathrm{EN}=$ endoderme; $\mathrm{EP}=$ epiderme; $\mathrm{EV}=$ elemento de vaso; $\mathrm{FE}=$ felogênio; $\mathrm{PE}=$ periciclo; $\mathrm{PX}=$ pólos de protoxilema; setas $=$ pêlos radiculares). Barra $=200 \mu \mathrm{m}(4,6), 50 \mu \mathrm{m}(5,8), 20 \mu \mathrm{m}(7)$.

Figures 4-8. Transversal sections of primary root of Pachyrhizus ahipa with seven days. 4. About $0.5 \mathrm{~cm}$ from the apex. 5. Detail of preview figure. 6 . About $2.2 \mathrm{~cm}$ from the apex. 7. Detail of the root hairs. 8. Detail of figure $6 .(\mathrm{CO}=$ cortex; $\mathrm{CV}=$ vascular cambium; $\mathrm{CV}^{\prime}$ = vascular cylinder; $\mathrm{EN}=$ endodermis; $\mathrm{EP}=$ epidermis; $\mathrm{EV}=$ vessel element; $\mathrm{FE}=$ phellogen; $\mathrm{PE}=$ pericycle; $\mathrm{PX}=$ poles of protoxylem; arrows $=$ root hairs $) . \mathrm{Bar}=200 \mu \mathrm{m}(4,6), 50 \mu \mathrm{m}(5,8), 20 \mu \mathrm{m}(7)$. 
vaso (figura 8). Ao mesmo tempo, verifica-se a formação do felogênio a partir de divisões, predominantemente, periclinais das células do periciclo (figura 8).

A raiz de Pachyrhizus ahipa com 17 dias (figuras $9,10)$, apresenta crescimento secundário e um câmbio de aspecto circular. Nesta fase, a epiderme ainda se mantém intacta, embora já se observe o surgimento do felogênio a partir das camadas externas do periciclo (figura 10). O córtex apresenta células de formato variado, observando-se o aparecimento de grandes espaços devido ao rompimento de algumas células parenquimáticas (figura 9).

$\mathrm{Na}$ medida em que a atividade cambial continua, observa-se na região mediana da raiz de $P$. ahipa, com 35 dias (figura 11), a presença de raios parenquimáticos largos, de origem do câmbio pericíclico, no xilema secundário. A epiderme e o córtex foram descartados e o felogênio produziu súber e, internamente, células parenquimáticas que apresentam conteúdo corado e constituem a feloderme (figura 11). Na região do floema primário observa-se a formação de fibras.

Aos 60 dias (figura 12), verifica-se o início do processo de tuberização da raiz devido à produção de uma quantidade muito maior de células parenquimáticas pelo câmbio. Essas células parenquimáticas, com tamanhos e formas variados, constituem o parênquima de reserva da raiz tuberosa.

Aos 90 dias (figura 13), na mesma região, observase o aumento de diâmetro da raiz tuberosa, devido não só à atividade do câmbio típico como, também, à atividade de câmbios acessórios que surgem a partir da retomada de divisão celular do parênquima do xilema secundário, de maneira concêntrica, após a primeira fase de crescimento secundário usual. Grupos esparsos de fibras gelatinosas ocorrem entre as células do parênquima de reserva.

Aos 120 dias, nota-se que a atividade dos câmbios acessórios é bastante intensa (figuras 14, 15) produzindo, predominantemente, parênquima mas, também, elementos floemáticos ou elementos xilemáticos e floemáticos.

A raiz de Pachyrhizus ahipa, com 150 dias (figura 16), apresenta estrutura semelhante à fase anterior, observando-se, porém, maior quantidade de parênquima de reserva, bem como de amido acumulado no interior destas células.

A região da raiz tuberosa, que não sofre tuberização, mostra estrutura secundária típica, com câmbio circular produzindo uma quantidade muito maior de elementos condutores em relação ao parênquima (figura 17). O felogênio, à semelhança do que ocorre na região tuberizada, forma-se a partir de células do periciclo, levando à eliminação da epiderme e camadas do córtex, à medida que a periderme se desenvolve (figura 17). Desenvolvimento do xilopódio - O xilopódio se forma a partir do crescimento secundário do hipocótilo e da região basal da raiz primária tuberosa.

Secções transversais seriadas, do hipocótilo de plantas de Pachyrhizus ahipa com 7, 12 e 17 dias, realizadas desde o nó cotiledonar até a região proximal da raiz primária, mostram estrutura típica de região de transição, com a torção dos pólos de protoxilema (figuras $18,19)$.

Na região próxima ao nó cotiledonar, o hipocótilo (figura 18) apresenta epiderme unisseriada e córtex amplo, composto por células parenquimáticas que apresentam formato e tamanho variados. $\mathrm{O}$ cilindro vascular apresenta aspecto quadrangular e, nesta fase, verifica-se a formação do felogênio a partir da divisão de células do periciclo (figura 18).

Secções transversais do hipocótilo, próximas à região basal da raiz primária, mostram um cilindro vascular com aspecto tendendo ao losangular, e pólos de protoxilema em posição exarca e endarca (figura 19). Nessa região também se observa a formação de raízes laterais (figura 19).

Aos 60 dias, observa-se que, em regiões próximas à raiz primária, o órgão apresenta estrutura típica de região de transição, com inversão dos pólos de protoxilema (figura 20). Posteriormente, nota-se um crescimento secundário decorrente da atividade do câmbio típico e de câmbios acessórios (figura 21), como observado na raiz primária tuberosa.

O xilopódio de plantas adultas de Pachyrhizus ahipa, com 150 dias (figura 22), em secção transversal próxima ao nó cotiledonar, apresenta câmbio circular que produz grande quantidade de xilema secundário. Nessa região verifica-se, também, o desenvolvimento de gemas caulinares (figura 22). As secções transversais, próximas à raiz primária tuberosa, apresentam estrutura semelhante àquela observada na mesma região do xilopódio com 60 dias (figura 23).

\section{Discussão}

Com base nos resultados obtidos e na literatura disponível, verifica-se que a terminologia mais adequada para a caracterização do sistema subterrâneo de Pachyrhizus ahipa é "xilopódio" para a porção superior (próxima ao solo) e raiz tuberosa, 

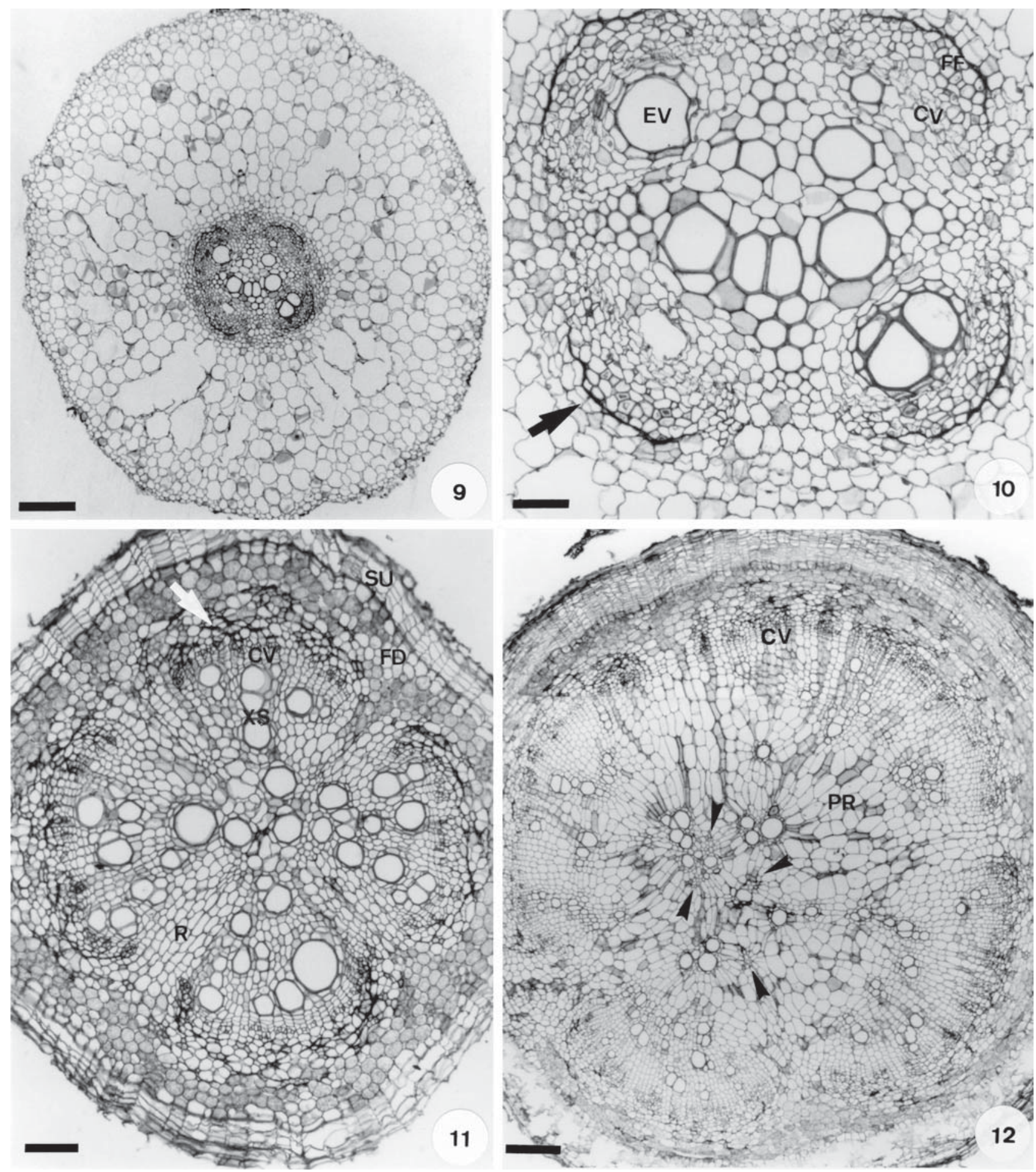

Figuras 9-12. Secções transversais medianas da raiz primária de Pachyrhizus ahipa. 9. Aspecto geral da raiz com 17 dias. 10. Detalhe da figura anterior, a seta indica o felogênio em início de formação. 11. Aspecto geral da raiz com 35 dias, a seta indica fibras do protofloema em início de formação. 12. Aspecto geral da raiz com 60 dias. (CV = câmbio vascular; $\mathrm{EV}=$ elemento de vaso; $\mathrm{FD}=$ feloderme; $\mathrm{FF}$ = fibras floemáticas; $\mathrm{PR}=$ parênquima de reserva; $\mathrm{R}=$ raio; $\mathrm{SU}=$ súber; $\mathrm{XS}=$ xilema secundário; pontas de seta $=$ pólos de protoxilema). Barra $=200 \mu \mathrm{m}(9,12), 100 \mu \mathrm{m}(11), 50 \mu \mathrm{m}(10)$.

Figures 9-12. Transversal sections of primary root of Pachyrhizus ahipa. 9. General view of the root with 17 days. 10. Detail of previous figure. 11. General view of the root with 35 days. 12. General view of the root with 60 days. $(\mathrm{CV}=$ vascular cambium; $\mathrm{EV}=$ vessel element; $\mathrm{FD}=$ pheloderm; $\mathrm{FF}=$ phloematic fibers; $\mathrm{PR}=$ storage parenchyma; $\mathrm{R}=$ ray; $\mathrm{SU}=$ cork; $\mathrm{XS}=$ secondary xylem; arrow-heads $=$ poles of protoxylem). Bar $=200 \mu \mathrm{m}(9,12), 100 \mu \mathrm{m}(11), 50 \mu \mathrm{m}(10)$. 

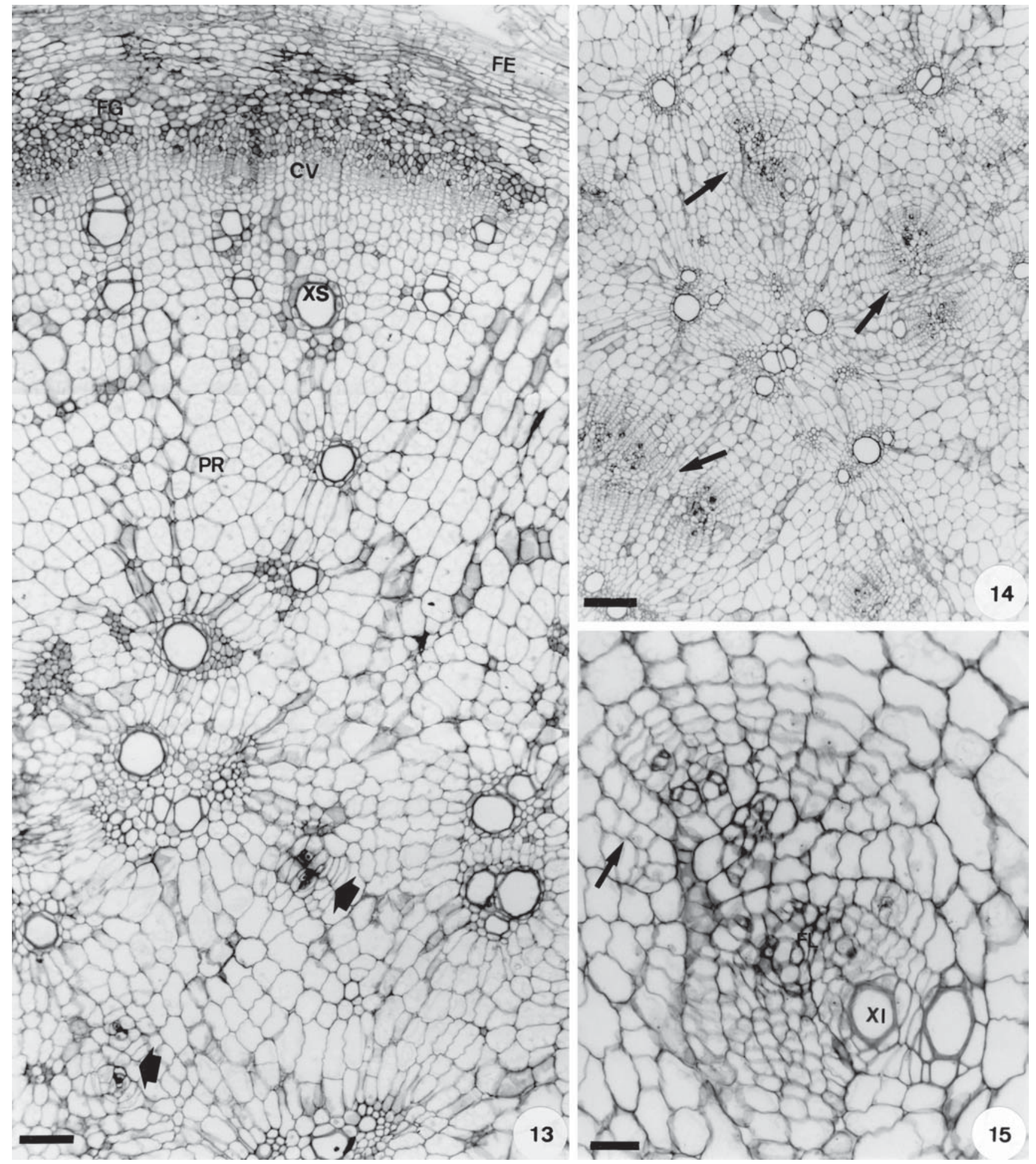

Figuras 13-15. Secções transversais medianas da raiz primária tuberosa de Pachyrhizus ahipa. 13. Aspecto geral da raiz com 90 dias. 14. Raiz com 120 dias. 15. Detalhe da figura anterior. $(C V=$ câmbio vascular; $F E=$ felogênio; $F L=$ floema; $P R=$ parênquima de reserva; $\mathrm{XI}=$ xilema; $\mathrm{XS}=$ xilema secundário; setas = câmbios acessórios; FG = fibras gelatinosas). Barra $=200 \mu \mathrm{m}(14)$, $100 \mu \mathrm{m}(13), 50 \mu \mathrm{m}(15)$.

Figures 13-15. Transversal sections of primary tuberous root of Pachyrhizus ahipa. 13. General view of the root with 90 days. 14. Root with 120 days. 15. Detail of previous figure. ( $\mathrm{CV}=$ vascular cambium; $\mathrm{FE}=$ phellogen; $\mathrm{FL}=$ phloem; $\mathrm{PR}=$ storage parenchyma; $\mathrm{XI}=$ xylem; $\mathrm{XS}=$ secondary xylem; arrows $=$ acessory cambium; FG = gelatinous fibers $) . \mathrm{Bar}=200 \mu \mathrm{m}(14), 100 \mu \mathrm{m}$ (13), $50 \mu \mathrm{m}(15)$. 
para a porção subjacente ao xilopódio.

Dos trabalhos analisados, pode-se constatar que é unânime a opinião dos pesquisadores a respeito da complexidade da estrutura dos sistemas subterrâneos, em especial do xilopódio. A primeira descrição de xilopódio (literalmente: "pé-de-madeira") foi realizada por Lindman (1906) que caracterizou a estrutura como um tipo de caule subterrâneo túbero-lignificado, comum nos terrenos pedregosos dos campos subarbustivos do Rio Grande do Sul.
As descrições realizadas por Lindman (1906), Rachid (1947), Rizzini (1965), Rizzini \& Heringer (1961, 1966) e os estudos mais recentes elaborados por Paviani (1978), Paviani \& Haridasan (1988) e Appezzato-daGlória \& Estelita (2000) relatam que os xilopódios têm como características comuns a capacidade gemífera, a consistência extremamente rígida e a anatomia complexa, podendo ser caulinar, radicular ou mista.

Segundo Paviani (1978), em Brasilia sickii G.M. Barroso (Asteraceae), o xilopódio deve ser considerado
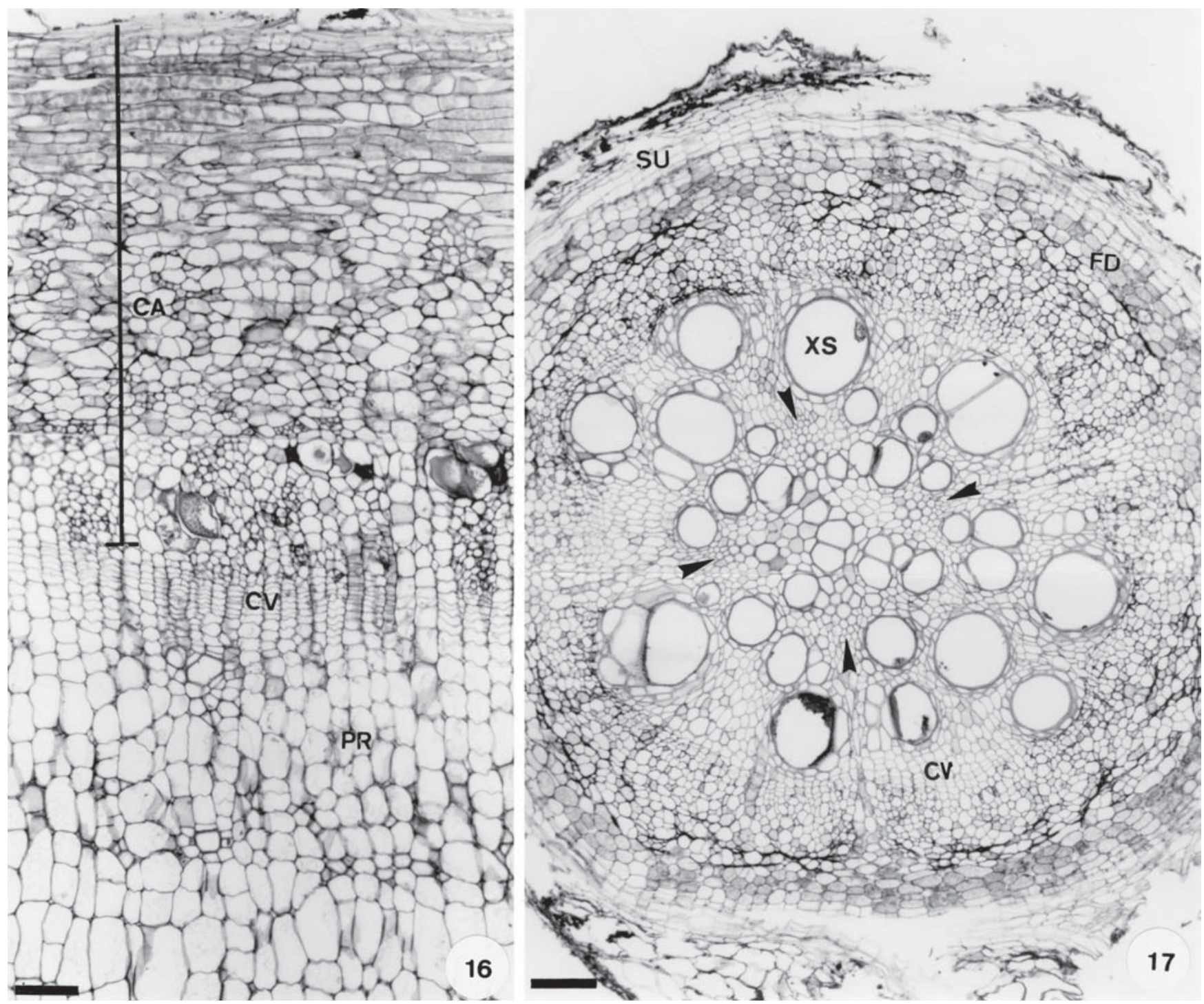

Figuras 16-17. Secções transversais medianas da raiz primária tuberosa de Pachyrhizus ahipa com 150 dias. 16. Aspecto geral da raiz. 17. Região não tuberizada da raiz primária. $(\mathrm{CA}=$ casca; $\mathrm{CV}=$ câmbio vascular; $\mathrm{FD}=$ feloderme; $\mathrm{PR}=$ parênquima de reserva; $\mathrm{SU}=$ súber; $\mathrm{XS}=$ xilema secundário; pontas de seta $=$ pólos de protoxilema). Barra $=100 \mu \mathrm{m}$.

Figures 16-17. Transversal sections of primary tuberous root of Pachyrhizus ahipa with 150 days. 16. General view of the root. 17. Non-tuberous root region. $(\mathrm{CA}=$ bark; $\mathrm{CV}=$ vascular cambium; $\mathrm{FD}=$ pheloderm; $\mathrm{PR}=$ storage parenchyma; $\mathrm{SU}=$ cork; $\mathrm{XS}=$ secondary xylem; arrow-heads $=$ poles of protoxylem). $\mathrm{Bar}=100 \mu \mathrm{m}$. 

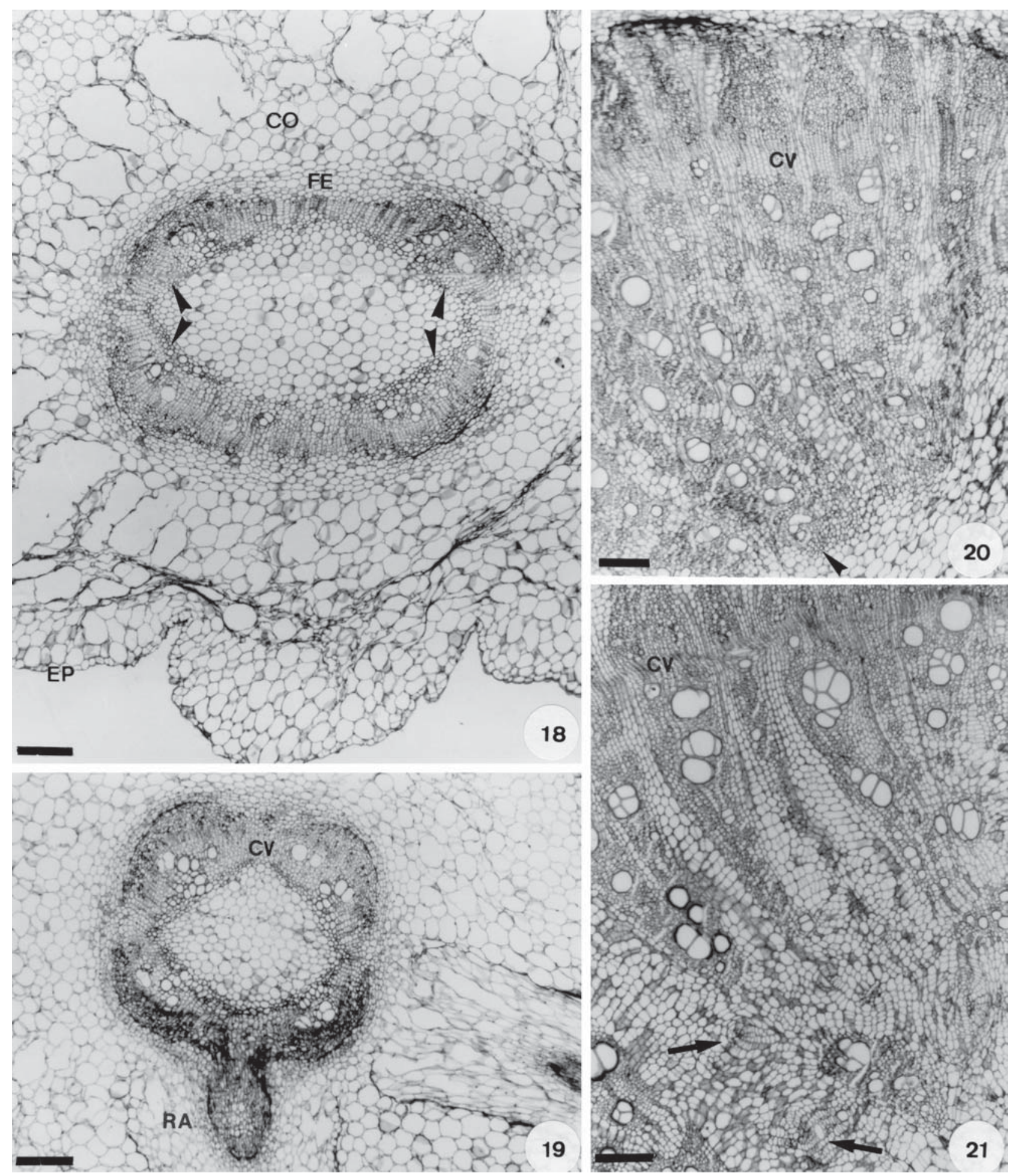

Figuras 18-21. Secções transversais do hipocótilo de Pachyrhizus ahipa. 18-19. Aspecto geral com 17 dias. 18. Próximo ao nó cotiledonar. 19. Próximo à região basal da raiz primária. 20-21. Aspecto geral com 60 dias. 20. Região de transição. 21. Próximo à região basal da raiz primária. $(\mathrm{CO}=$ córtex; $\mathrm{CV}=$ câmbio vascular; $\mathrm{EP}=$ epiderme; $\mathrm{FE}=$ felogênio; $\mathrm{RA}=$ raiz adventícia; pontas de seta $=$ pólos de protoxilema; setas $=$ câmbios acessórios $)$. Barra $=200 \mu \mathrm{m}$.

Figures 18-21. Transversal sections of hypocotyl of Pachyrhizus ahipa. 18-19. General view with 17 days. 18. Near from cotyledon node. 19. Near from the base of the primary root. 20-21. General view with 60 days. 20. Transition region. 21. Near from the base of the primary root. $(\mathrm{CO}=$ cortex; $\mathrm{CV}=$ vascular cambium; $\mathrm{EP}=$ epidermis; $\mathrm{FE}=$ phellogen; $\mathrm{RA}=$ adventiceous root; arrow-heads $=$ poles of protoxylem; arrows $=$ acessory cambium $).$ Bar $=200 \mu \mathrm{m}$. 

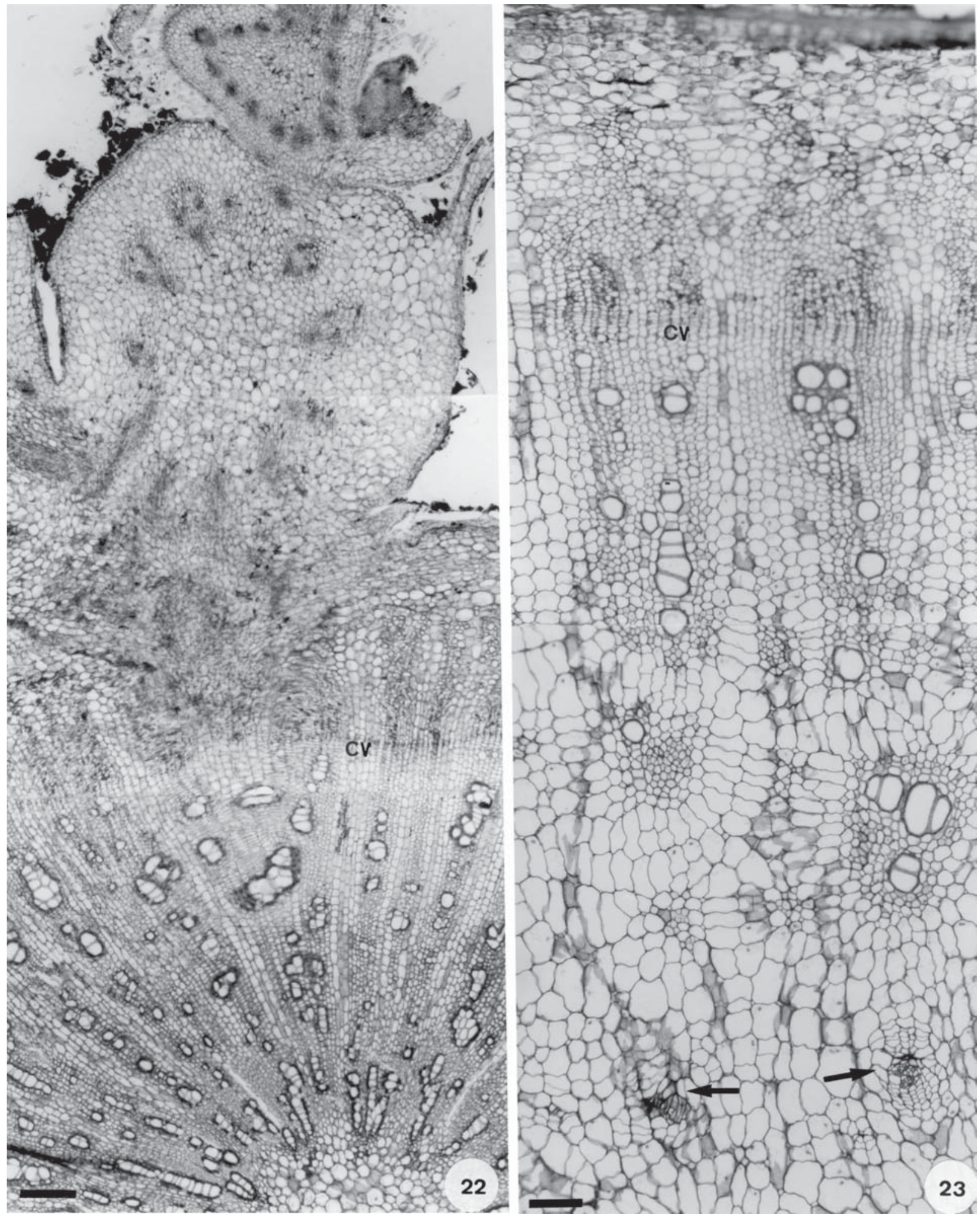

Figuras 22-23. Secções transversais do xilopódio de Pachyrhizus ahipa com 150 dias. 22. Aspecto geral, próximo ao nó cotiledonar. 23. Aspecto geral, próximo à região basal da raiz tuberosa. $(\mathrm{CV}=$ câmbio vascular; setas = câmbios acessórios). Barra $=200 \mu \mathrm{m}(22), 100 \mu \mathrm{m}(23)$.

Figures 22-23. Transversal sections of xylopodium of Pachyrhizus ahipa with 150 days. 22. General view near from cotyledon node. 23 . General view near from the base of the tuberous root $(\mathrm{CV}=$ vascular cambium; arrows = acessory cambium $)$. $\mathrm{Bar}=$ $200 \mu \mathrm{m}(22), 100 \mu \mathrm{m}(23)$. 
uma unidade morfológica e não anatômica, por apresentar estrutura ora caulinar (quando novo), ora radicular (quando adulto).

Appezzato-da-Glória \& Estelita (2000) relataram que em Mandevilla illustris (Vell.) Woodson e M. velutina (Mart. ex Standelm.) Woodson (Apocynaceae), o xilopódio apresenta estrutura predominantemente caulinar, sendo originado do hipocótilo, embora, em alguns espécimes, tenham verificado que o xilopódio apresenta estrutura mista (caulinar/radicular), formando-se a partir do hipocótilo e da região basal da raiz primária.

A análise ontogenética realizada no presente trabalho permitiu observar que os resultados obtidos sobre a formação do xilopódio de Pachyrhizus ahipa corroboram com as descrições de Paviani (1978) e Appezzato-da-Glória \& Estelita (2000).

Até o momento, não foram localizados trabalhos que relacionassem diretamente o tipo de germinação às estruturas formadoras do xilopódio. Entretanto, Rizzini (1965) verificou que quando o tipo de germinação é hipógea, não havendo hipocótilo individualizado, o xilopódio forma-se a partir da porção superior da raiz primária. Já, em espécies como Mimosa multipina Benth., onde a germinação é epígea, o xilopódio origina-se do hipocótilo e da porção superior da raiz primária. Em $P$. ahipa, o tipo de germinação é semi-hipógea e o xilopódio se origina do hipocótilo e da raiz primária não corroborando com o observado por Rizzini (1965).

Segundo Rizzini \& Heringer (1961) o xilopódio não deve ser considerado um órgão tipicamente de reserva, assim como são as raízes tuberosas. Já, Rawitscher \& Rachid (1946) referem-se aos xilopódios como órgãos ricos em água e substâncias de reserva.

Paviani (1974) relata que o xilopódio de Brasilia sickii é, sobretudo, uma estrutura de armazenamento de água e sugere que esta reserva ocorra, principalmente, nas paredes das fibras gelatinosas.

Os resultados obtidos permitem sugerir que o xilopódio de $P$. ahipa representa uma estrutura armazenadora de água, devido à presença de fibras gelatinosas em grande quantidade e indicam, também, que se trata de uma estrutura de reserva devido à presença de amido no parênquima do xilema, não se observando, no entanto, a ocorrência de um parênquima fundamentalmente de reserva (amido) como verificado na raiz tuberosa. Este fato está de acordo com as idéias de Rizzini \& Heringer (1961) e Paviani (1974).

Das considerações realizadas por Rizzini \& Heringer (1961) e Rizzini $(1963,1965)$ sobre a formação e o desenvolvimento do xilopódio, observa-se que em algumas espécies o xilopódio representa um fator geneticamente determinado, surgindo em quaisquer meios em que a planta possa crescer, ao passo que em outras, a formação do xilopódio é determinada pela interação do fator genético com fatores ambientais.

Em Mimosa multipina, a formação do xilopódio é diretamente dependente do fator edáfico (alta compacidade do solo). Neste caso, o clima exerce papel secundário, favorecendo ou retardando o desenvolvimento do xilopódio (Rizzini 1963). Por outro lado, Paviani (1977) verificou que o xilopódio de Brasilia sickii é geneticamente determinado, pois observou o início de sua formação, já em plântulas de 2 meses.

As observações realizadas durante o desenvolvimento de Pachyrhizus ahipa, tanto no campo, quanto em casa-de-vegetação não permitiram estabelecer qual é o fator responsável pela formação do xilopódio desta espécie, sendo necessário um estudo capaz de analisar os diferentes fatores que possam favorecer, retardar ou mesmo impedir a formação do xilopódio, ou ainda, constatar a determinação genética desse órgão.

Em relação às raízes de Leguminosae, Compton (1912) relata que nas raízes primárias é freqüente o padrão tetrarco, após a diferenciação dos tecidos vasculares primários. Em Phaseoleae, as raízes primárias são triarcas, sendo comum o desenvolvimento de um vaso de metaxilema central (Lackey 1981). Bianchini \& Corso (1992) verificaram que a raiz primária de plântulas de Stizolobium aterrimum Piper \& Tracy (Phaseoleae) apresenta estrutura tetrarca, com metaxilema ocupando a posição central. Em Pisum sativum L. as raízes apresentam organização triarca, sendo raramente tetrarcas (Hayward 1953). Os dados para raiz de $P$. ahipa estão de acordo com as descrições feitas por Compton (1912), mas não condizem com o relatado por Lackey (1981) para a tribo Phaseoleae.

De acordo com Eames \& MacDaniels (1947), Esau (1965) e Fahn (1990), na maioria das espécies, o número de pólos de protoxilema, no cilindro central da raiz, pode variar entre plantas diferentes ou em raízes diferentes de uma mesma planta.

Gabrielli (1992) verificou, para Pyrostegia venusta (Ker) Miers, uma variação na organização do estelo da raiz, que pode ser triarco a heptarco. Situação semelhante foi relatada por Appezzato-da-Glória \& Estelita (2000) para as raízes de Mandevilla illustris e $M$. velutina, cuja organização do estelo varia de triarco a hexarco. No presente trabalho, verificou-se que o padrão de organização tetrarco da raiz de Pachyrhizus ahipa é constante. 
Nas raízes, em geral, o periciclo é unisseriado podendo, em alguns casos, apresentar várias camadas de células (Esau 1965). Periciclo multisseriado foi observado na raiz primária de Medicago sativa L. (Leguminosae) (Hayward, 1953) e no presente trabalho, em Pachyrhizus ahipa.

Segundo Esau (1965), apesar das variações estruturais, todos órgãos de reserva apresentam grande quantidade de parênquima de reserva. Segundo a mesma autora, isso pode ocorrer pela proliferação de parênquima entre os elementos vasculares normalmente localizados ou, ainda, pelo desenvolvimento de novos sistemas vasculares, externamente ao cilindro vascular normalmente localizado.

Em Daucus carota L. (Esau 1940), Oxalis (Estelita-Teixeira 1982), Mandevilla illustris e M. velutina (Appezzato-da-Glória \& Estelita 2000) o processo de tuberização das raízes é resultante de um crescimento secundário comum. Porém, em inúmeras espécies, a tuberização decorre de um crescimento secundário não usual, conforme relatado por Artschwager (1924), Hayward (1953), Wilson \& Lowe (1973), Dabydeen \& Sirju-Charran (1990). Este tipo de crescimento, também, foi verificado na raiz tuberosa de Pachyrhizus ahipa.

Hayward (1953) verificou que o crescimento em espessura da raiz tuberosa de Beta vulgaris resulta da formação de várias camadas cambiais ("câmbios supernumerários"), dispostas concentricamente, externamente ao cilindro vascular normal, produzindo células xilemáticas, floemáticas e parênquima de reserva. Em Ipomoea batatas (L.) Lam. observa-se inicialmente, o surgimento de câmbios ao redor dos pólos de protoxilema e do metaxilema central e, posteriormente, a formação de câmbios ao redor de elementos de vaso isolados ou em grupo, produzindo grande quantidade de parênquima de reserva, elementos traqueais, elementos crivados e laticíferos (Artschwager 1924, Hayward 1953, Wilson \& Lowe 1973).

Dabydeen \& Sirju-Charran (1990) verificaram, em $P$. erosus, o surgimento de câmbios a partir do parênquima do xilema secundário e de tecidos produzidos pelos mesmos. Este padrão foi observado na raiz tuberosa de Pachyrhizus ahipa, verificando-se ainda, que o parênquima produzido acumula grande quantidade de amido.

Agradecimentos - À Fundação de Amparo á Pesquisa do Estado de São Paulo (Fapesp), pela bolsa de Mestrado (Processo n. 00/05657-8) e ao Centro de Estudos de Raízes e Amidos Tropicais (Cerat), pelo fornecimento das sementes.

\section{Referências bibliográficas}

APPEZZATO-DA-GLÓRIA, B. \& ESTELITA, M.E.M. 2000. The developmental anatomy of the subterranean system in Mandevilla illustris (Vell.) Woodson and M. velutina (Mart. ex Stadelm.) Woodson (Apocynaceae). Revista Brasileira de Botânica 23:27-35.

ARTSCHWAGER, E. 1924. On the anatomy of the sweet potato root with notes on internal breakdown. Journal of Agricultural Research 27:157-166.

BIANCHINI, E. \& CORSO, G.M. 1992. Anatomia de plântulas de Stizolobium aterrimum Piper et Tracy (Leguminosae) - mucuna preta. Anais da Academia Brasileira de Ciências 64:305-315.

COMPTON, R.H. 1912. Investigation of the seedling structure in the Leguminosae. Journal of the Linnean Society of London Botany 41:1-122.

DABYDEEN, S. \& SIRJU-CHARRAN, G. 1990. The developmental anatomy of the root system in yam bean, Pachyrhizus erosus Urban. Annals of Botany 66:313-320.

EAMES, A.J. \& MAC DANIELS, L.H. 1947. An introduction to plant anatomy. McGraw-Hill, New York.

ESAU, K. 1940. Developmental anatomy of the fleshy storage organ of Daucus carota. Hilgardia 13:175-226.

ESAU, K. 1965. Plant anatomy. John Wiley, New York.

ESTELITA-TEIXEIRA, M.E. 1982. Shoot anatomy three bulbous species of Oxalis. Annals of Botany 49:805-813.

FAHN, A. 1990. Plant anatomy. $4^{\text {th }}$ ed. Pergamon Press, Oxford.

FORSYTH, J.L., RING, S.G., NOEL, T.R., PARKER, R., CAIRNS, P., FINDLAY, K. \& SHEWRY, P.R. 2002. Characterization of starch form tubers of yam bean (Pachyrhizus ahipa). Journal of Agricultural and Food Chemistry 50:361-367.

GABRIELLI, A.C. 1992. Contribuição ao estudo anatômico de Pyrostegia venusta (Ker) Miers - Bignoniaceae. Revista Brasileira de Botânica 15:95-104.

GERRITS, P.O. 1964. The application of glycol metacrylate histotechnology: some fundamental principles. Leica Gmbh, Germany.

GRAU, A. 1997. Ahipa, la legumbre tuberose de los Andes. Ciencia Hoy 7:31-38.

HANSBERRY, R., CLAUSEN, R.T. \& NORTON, L.B. 1947. Variations in the chemical composition and insecticidal properties of the yam bean (Pachyrhizus). Journal of Agricultural Research 74:55-64.

HAYWARD, D.H. 1953. Estrutura de las plantas utiles. Acme, Buenos Aires.

JOHANSEN, D.A. 1940. Plant microtechnique. McGraw-Hill, New York.

KAY, D.E. 1973. Yam bean, potato bean. In Root crops (D.E. Kay, ed.). Tropical Products Institute, London, p.240-245.

KJAER, S. 1992. Biological nitrogen fixation in Pachyrhizus ahipa (Wedd.) Parodi. Annals of Botany 70:11-17. 
LACKEY, J.A. 1977. A revised classification of the tribe Phaseoleae (Leguminosae: Papilionioideae) and its relation to canavanine distribution. Botanical Journal of the Linnean Society 74:163-178.

LACKEY, J.A. 1981. Phaseoleae DC. (1825). In Advances in legume systematics (R.M. Polhill \& P.H. Raven, eds.). Royal Botanic Gardens, Kew, p.301-327.

LINDMAN, C.A.M. 1906. A vegetação no Rio Grande do Sul. Trad. A. Löfgren. Livraria Universal, Porto Alegre.

O'BRIEN, T.P., FEDER, N. \& MCCULLY, M.E. 1964. Polychromatic staining of plant cell walls by toluidine blue O. Protoplasma 59:368-373.

PAVIANI, T.I. 1974. Sobre a ocorrência de fibras gelatinosas em Plathymenia reticulata Benth. Ciência e Cultura 26:783-786.

PAVIANI, T.I. 1977. Estudo morfológico e anatômico de Brasilia sickii G.M. Barroso; II: Anatomia da raiz, do xilopódio e do caule. Revista Brasileira de Botânica 37:307-324.

PAVIANI, T.I. 1978. Anatomia vegetal e cerrado. Ciência e Cultura 30:1076-1086.

PAVIANI, T.I. \& HARIDASAN, M. 1988. Tuberosidade em Vochysia thyrsoidea Pohl (Vochysiaceae). Ciência e Cultura 40:998-1003.

RACHID, M. 1947. Transpiração e sistemas subterrâneos da vegetação de verão do Campos Cerrados de Emas. Boletim da Faculdade de Filosofia Ciências e Letras da Universidade de São Paulo 5:5-140.

RAWITSCHER, F.K. \& RACHID, M. 1946. Troncos subterrâneos de plantas brasileiras. Anais da Academia Brasileira de Ciências 18:261-280.
RIZZINI, C.T. 1963. O fator edáfico na formação do xilopódio de Mimosa multipinna Benth. Anais da Academia Brasileira de Ciências 35:75-77.

RIZZINI, C.T. 1965. Estudos experimentais sobre o xilopódio e outros órgãos tuberosos de plantas do Cerrado. Anais da Academia Brasileira de Ciências 37:87-113.

RIZZINI, C.T. \& HERINGER, E.P. 1961. Underground organs of plants from some southern Brasilian savannas, with special reference to the xylopodium. Phyton 17:105-124.

RIZZINI, C.T. \& HERINGER, E.P. 1966. Estudo sobre os sistemas subterrâneos difusos de plantas campestres. Anais da Academia Brasileira de Ciências 38:85-112.

ROCHA, D.C. 1995. Estudos morfoanatômicos dos órgãos vegetativos de Dioscorea kunthiana Uline ex R. Knuth (Dioscoreaceae). Dissertação de mestrado, Universidade de São Paulo, São Paulo.

SØRENSEN, M. 1988. A taxonomic revision of the genus Pachyrhizus (Fabaceae - Phaseolae). Nordic Journal of Botany 8:167-192.

SØRENSEN, M. 1990. Observations on distribution, ecology and cultivation of the tuber-bearing legume genus Pachyrhizus Rich. ex DC. Wageningen Agricultural University Papers 90:1-38.

SØRENSEN, M., GRUNEBERG, W.J. \& ØRTING, B. 1997. Ahipa (Pachyrhizus ahipa (Wedd.) Parodi). In Andean roots and tubers: ahipa, arracacha, maca and yacon. Promoting the conservation and use of underutilized and neglected crops (M. Herman \& J. Heller, eds.). International Plant Genetic Resources Institute, Rome, p.13-73.

WILSON, L.A. \& LOWE, S.B. 1973. The anatomy of the root system in West Indian sweet potato (Ipomoea batatas (L.) Lam.) cultivars. Annals of Botany 37:633-643. 
